\title{
Testing of an End-Point Control Unit Designed to Enable Precision Control of Manipulator-Coupled Spacecraft
}

\author{
Raymond C. Montgomery and Dave Ghosh \\ Spacecraft Controls Branch \\ NASA Langley Research Center \\ Hampton, Virginia 23681
}

Patrick A. Tobbe and John M. Weathers

Logicon Control Dynamics, Inc. Huntsville, Alabama 35802

Davoud Manouchehri and Thomas S. Lindsay

Rockwell Space Systems Division

Downey, California 90241

\author{
AIAA Space Programs and \\ Technologies Conference and Exhibit
}

September 27-29, 1994

Huntsville, Alabama 


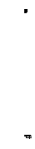




\title{
TESTING OF AN END-POINT CONTROL UNIT DESIGNED TO ENABLE PRECISION CONTROL OF MANIPULATOR-COUPLED SPACECRAFT
}

\author{
Raymond C. Montgomery", Dave Ghosh ${ }^{\dagger}$, \\ Patrick A. Tobbe $\ddagger$, John M. Weathers $\$$, \\ Davoud Manouchehri", and Thomas S. Lindsay"
}

Dynamics and Control Branch

NASA Langley Research Center

Hampton, VA 23666

Abstract

This paper presents an end-point control concept designed to enable precision telerobotic control of manipulator-coupled spacecraft. The concept employs a hardware unit (end-point control unit, EPCU) that is positioned between the end-effector of the Space Shuttle Remote Manipulator System and the payload. Features of the unit are active compliance (control of the displacement between the end-effector and the payload), to allow precision control of payload motions, and inertial load relief, to prevent the transmission of loads between the end-effector and the payload. This paper presents the concept and studies the active compliance feature using a simulation and hardware. Results of the simulation show the effectiveness of the EPCU in smoothing the motion of the payload. Results are presented from initial, limited tests of a laboratory hardware unit on a robotic arm testbed at the Marshall Space Flight Center. Tracking performance of the arm in a constant speed automated retraction and extension maneuver of a heavy payload with and without the unit active is compared for the design speed and higher speeds. Simultaneous load reduction and tracking performance are demonstrated using the EPCU.

- Aero-Space Technologist, NASA Langley Research Center, Hampton, Virginia, Associate Fellow, AIAA

$\dagger \quad$ Principal Engineer, Lockheed Engineering and Sciences Co., Hampton, Virginia, Associate Fellow, AIAA

* Manager, Space Systems Division, Logicon Control Dynamics, Inc. Huntsville, Ala., Member, AIAA

$\$$ Staff Engineer, Logicon Control Dynamics, Inc. Huntsville, Ala.

- Senior Engineering Specialist, Rockwell Space Systems Division, Downey, CA

- MTS, Automation and Robotics, Rockwell Space Systems Division, Downey, CA
I. Introduction

The capture and berthing of a controlled spacecraft using a robotic manipulator is an important technology for future space missions and is presently planned for early Space Station assembly missions. The capture and berthing operation requires precision telerobotic maneuvering of large payloads by the astronauts using the Remote Manipulator System (RMS) of the Space Shuttle. Since the RMS was not designed with this level of precision in mind, augmentation of the system may be needed. The problem of concern is controlling the motions of large payloads during start-up and stopping. During these operations, the direction of motion of the payload is difficult to predict because of start-up transients and the vibration environment of the system. Augmentation that will allow precision payload positioning and active monitoring of loads transmitted to the payload is desirable. A requirement is that the augmentation should solve the problem with little or no impact on the existing RMS. The potential solution proposed herein involves an independent device called an End-Point Control Unit (EPCU) that interfaces the payload and the end-effector of the RMS and actively controls loads transmitted to the payload using active compliance and/or inertial load relief.

The EPCU is positioned between the end of a manipulator and the payload and provides vibration isolation through active compliance and/or inertial load relief using inertia devices such as control moment gyros (CMGs), reaction wheels, or reaction mass actuators. The EPCU would be a tool, stored in the payload bay until needed, picked up by the RMS or other compatible telerobotic manipulator, and used to grapple the payload. Astronaut inputs would be provided via a Power/Data Grapple Fixture (PDGF) which would return appropriate signals so that the astronaut can monitor safe operation of the unit.

Previous research in vibration control of manipulator arms with large payloads has been directed at using joint motors only [1]. To the authors' knowledge, the 
first proposal to use active inertial devices to control the vibrations of robot arms was by Timmerman and Dickerson [2]. In that paper, the concept was put forth and explained using a simple spring-mass system. Reference [3] provides a theoretical development and simulation study of a two-link manipulator arm wherein a torque-wheel device was evaluated for improving the performance of a robot arm. Therein, it was shown by simulation that the use of a torque-wheel could substantially reduce the overshoot occurring as a result of a sudden stop command from the operator. Thus, the device would be useful in suppressing vibrations at the payload end and generally in improving the operability of a manipulator arm.

An active inertial device that is positioned between the payload and the current end-effector of the RMS and provides motion and vibration isolation was proposed in [4]. Active compliance alone was also proposed as a potential solution to the problem in [5]. Therein, active compliance was investigated using a simulation of a hardware testbed available at the Marshall Space Flight Center [6]. The mechanism simulated used a direct drive motor mounted to the manipulator end of the device to drive a connecting rod attached to the payload end via a load cell. Thus, the load transmitted to the payload could be directly controlled via a high gain feedback loop from the load cell to the motor. This paper is, in fact, a continuation of that research and focuses on the initial tests conducted at the Marshall Space Flight Center. The paper presents a brief description of the EPCU concept and design, overviews the facility at MSFC [6] which has been used to test the device, describes a simulator for the facility with the EPCU installed, presents initial simulation results which show that the device has the potential of isolating the payload motions from vibrations of the arm, and presents results taken from the May 1994 test program at MSFC.

\section{EPCU Concept and Design}

The EPCU concept, applies to a broad range of devices (e.g. active compliance mechanisms, CMGs, reaction wheels, or reaction mass actuators) that interface between the end of a manipulator and the payload (see figure 1), and provide a precision position control capability together with vibration isolation (using active compliance) and/or inertial load relief. The device must generate motor commands, using the astronauts' hand controller, that produce smooth and predictable payload motions with little vibration. Because of the cost of modification of the existing RMS controller, the EPCU must be designed to as a stand-alone unit attached between the manipulator arm and its payload which uses existing RMS interfaces. Signals from EPCU mounted sensors as well as input from the astronaut's hand controllers are used to compute the actuation motion of the EPCU to improve its performance. Improved performance includes better ability of the payload to track commanded payload velocity, and the reduction of forces acting at the end of the manipulator.

One mode of operation is to lock the joints of the manipulator and use the device strictly for precision placement within its deflection limits. Another mode involves using the unit during large angle maneuvers of the manipulator to provide a controlled interface between the payload and the manipulator for load control and vibration isolation.

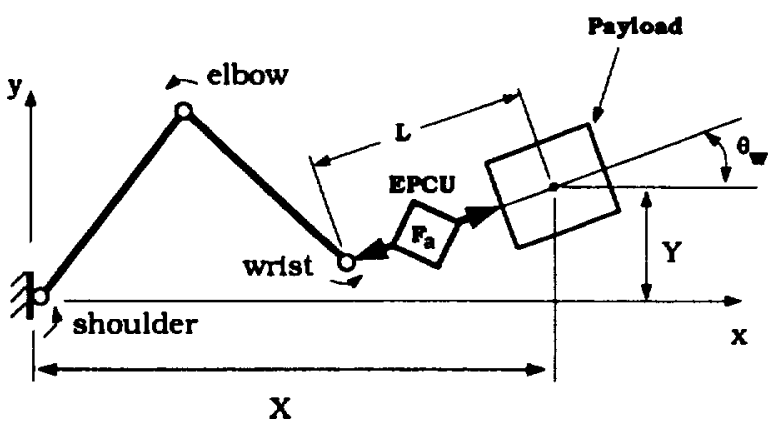

Figure 1.- EPCU concept and simulation nomenclature

The EPCU device tested in May, 1994 at the Marshall Space Flight Center has only one degree of freedom of actuation and is composed of four subcomponents: 1) the drive mechanism, 2) the linear motion constraints, 3) feedback sensors, and 4) digital control hardware and software. Figure 2 is a schematic diagram of the EPCU tested.

The drive mechanism consists of a motor controller and a 5-phase stepper motor with a $2.54 \mathrm{~cm}$ pitch diameter gear driving a rack, providing linear motion control. The motor controller outputs 1000 steps per revolution.

A round shaft riding in a linear bearing block and a pair of guide wheels running on linear rails constrain the relative motion of the EPCU to one degree of freedom. In this direction, the constraints allow motion of $\pm 2.54 \mathrm{~cm}$. in the precision positioning mode. 


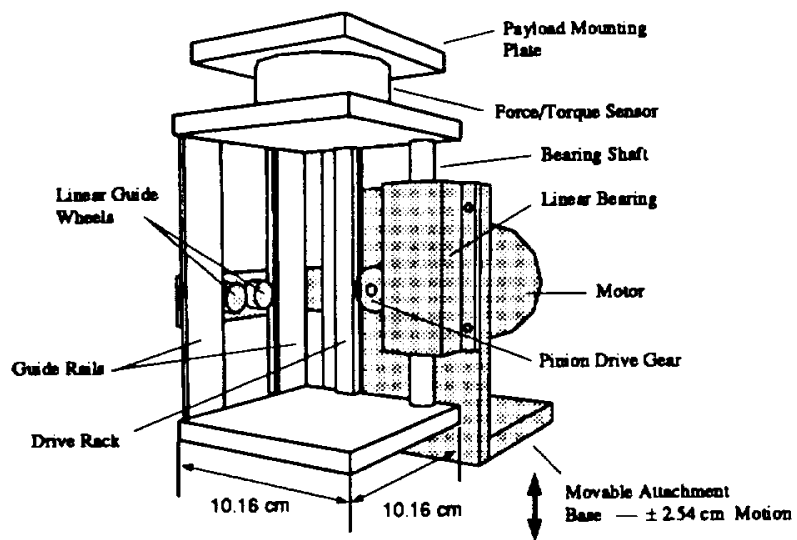

Figure 2.- A schematic diagram of the EPCU tested.

Feedback sensors include an encoder mounted directly to the motor shaft, and a 6-axis force/torque sensor. The encoder has 1500 counts per revolution, read-in quadrature, so that there is an effective resolution of 6000 counts per revolution. The force/torque sensor has a load limit of $448 \mathrm{~N}$ of force in the axis of motion, which is currently the only axis monitored.

The digital control hardware is a typical VME-bus based, laboratory computer system with a realtime operating system. It provides the capability of advanced control law implementation for the EPCU in the $C$ programming language, the system level interfaces to the EPCU sensors and motor controller as well as the control computer for the ManipulatorCoupled Spacecraft Testbed. The only signals provided in the interface to the testbed are a discrete digital signal for "run in progress" and three real time analog data channels interpreted as hand controller inputs from the testbed monitored by the EPCU. The digital control cycle is free running with a sample rate of approximately $160 \mathrm{cycles} / \mathrm{second}$.

\section{Manipulator-Coupled Spacecraft Testbed}

The experience in space for operations involving the Space Shuttle, the RMS, and payloads has been confined to payloads without active attitude control capability. Experience is needed in maneuvering active payloads with attitude control shared between both the payload and the handler. This would be the case of the berthing operation of the Space Shuttle to the Space Station. NASA Langley Research Center in cooperation with MSFC has assembled an evolutionary testbed to conduct research in the berthing operation and, in general, research into the control of multibody configurations that are loosely coupled with flexible manipulator linkages.

When completed, the configuration of the testbed is to be a model of the Space Shuttle and the Remote
Manipulator during berthing of a large spacecraft, e.g. the Space Station, constrained to move in the horizontal plane. Figure 3 illustrates the primary components of the completed testbed. Referring to the sketch: ABV1 (air bearing vehicle 1), representing the Space Station, has a mass of $1694.35 \mathrm{~kg}$. It is supported on the MSFC flat floor facility using low flow-rate air bearings. ABV1 also has cold gas reaction jets to allow translational and rotational maneuvering and a single gimbal CMG for attitude control. Onboard sensors include laser scanners that measure location of the vehicle on the floor, a rate gyro, and accelerometers for feedback control. The onboard control computer is a 486 -based machine which communicates with a fixed console off of the floor via an RF telecommunications link and provides a $20 \mathrm{~Hz}$ control loop. The other vehicle, referred to as $A B V 2$ in figure 3, representative of the Space Shuttle, is to be of similar construction and will be attached to $A B V 1$ via a three degree-of-freedom, two-link manipulator arm. The three joints of the arm are driven by electric motors, and the elbow joint is supported by air bearings. This testbed is planned to be operational in late summer, 1994.

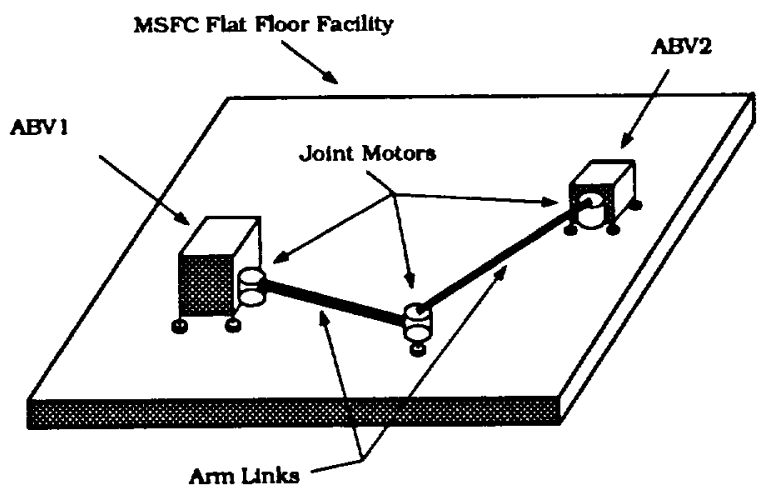

Figure 3.- Multibody controls research testbed.

To facilitate early experience, an interim testbed has been assembled using the ABV1 vehicle. This testbed consists of a two-link manipulator arm coupled to the vehicle with the shoulder joint attached to the wall of the flat floor facility (see figure 4). The arm linkage consists of two $2.74 \mathrm{~m}$ long aluminum I-beams each with $0.076 \mathrm{~m}$ by $0.0032 \mathrm{~m}$ flanges and $0.1 \mathrm{~m}$ by $0.0032 \mathrm{~m}$ webs. The links are connected to the joint motors using harmonic drive gear trains with a maximum torque output of $271 \mathrm{~N}-\mathrm{m}, 226 \mathrm{~N}-\mathrm{m}$, and $124 \mathrm{~N}-\mathrm{m}$ for the shoulder, elbow, and wrist joints. respectively.

\section{Simulation Description and Results}

LaRC has developed a simulator for the interim testbed using a commercially available simulation package [7]. Elements of the testbed are modelled as 


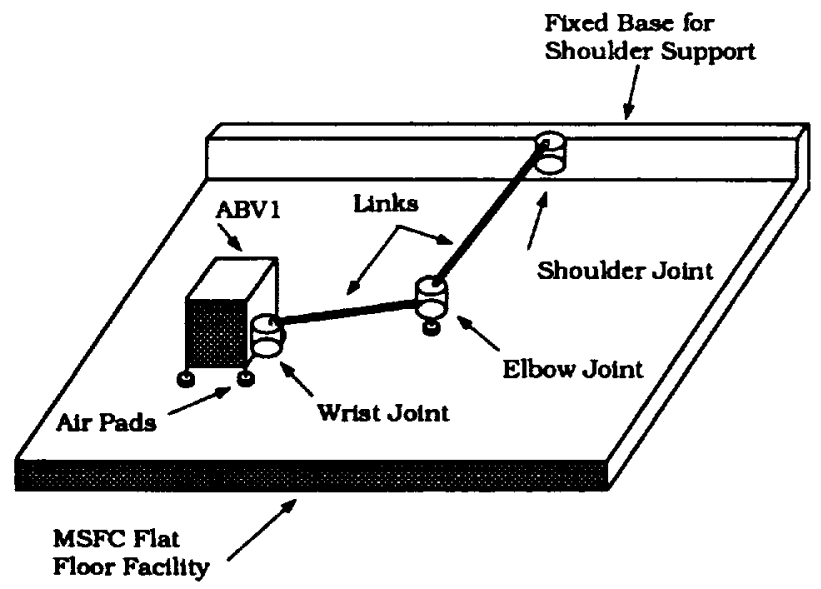

Figure 4.- Interim testbed.

rigid bodies. Flexibility is lumped into the models of the gear trains. The equations of motion for the testbed were developed using a Lagrangian formulation with a commercially available symbolic manipulator [8]. A complete description of the simulator is included in reference [6].

As installed on the testbed, the EPCU allows a single degree-of-freedom relative motion along a line of action between the payload, ABV1, and the wrist attachment point of the manipulator. Placement of the unit on the testbed is indicated in figure 1 which also shows the coordinate system and nomenclature used herein. The line of action makes an angle $\theta_{w}$ with respect to the $x$-coordinate axis. The coordinate $L$ is the relative displacement between the wrist and the payload center of gravity and is measured along the line of action of the EPCU. The force between the base of the EPCU and the payload is $F_{\mathrm{a}}$.

A simulation has been developed for the facility with the EPCU installed as shown in figure 1. It assumes that the torque and force normal to the line of action of the EPCU are directly transmitted to the vehicle but that the force along the line of action of the EPCU can be actively controlled. The simulation is, otherwise, the same as described in [6].

Simulation results are included herein that illustrate the vibration isolation feature of the EPCU. A proportional control law was used with a gain of 1000 $\mathrm{N} / \mathrm{m}$ on the axial force $\mathrm{F}_{\mathrm{a}}$ from the displacement $\mathrm{L}$. The initial conditions corresponded to the configuration shown in figure 5 and the excitation input was a square-wave-doublet torque-input to the wrist motor. Until time, $t$, of 2 sec., the input is zero. The wave doublet starts at time $t=2 \mathrm{sec}$. with an amplitude of $40 \mathrm{~N}-\mathrm{m}$ and switches to $-40 \mathrm{~N}-\mathrm{m}$ at $\mathrm{t}=4 \mathrm{sec}$. For $\mathrm{t} \geq 6$ sec. the input is zero. Figure 6 shows that the variation of axial force transmitted to ABV1 is $\pm 4 \mathrm{~N}$. The axial extension of the EPCU is shown in figure 7 and is about $10 \mathrm{~mm}$ peak to peak. Figures 8 and 9 show the planar motion of the wrist and ABV1, respectively.

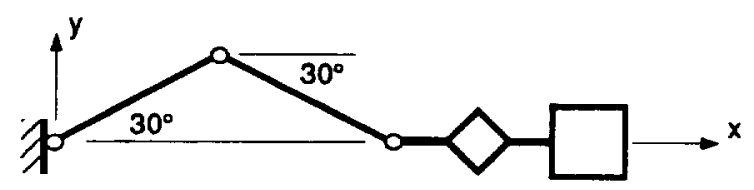

Figure 5.- Configuration of the arm selected for the simulation and initial configuration for experimental test runs.

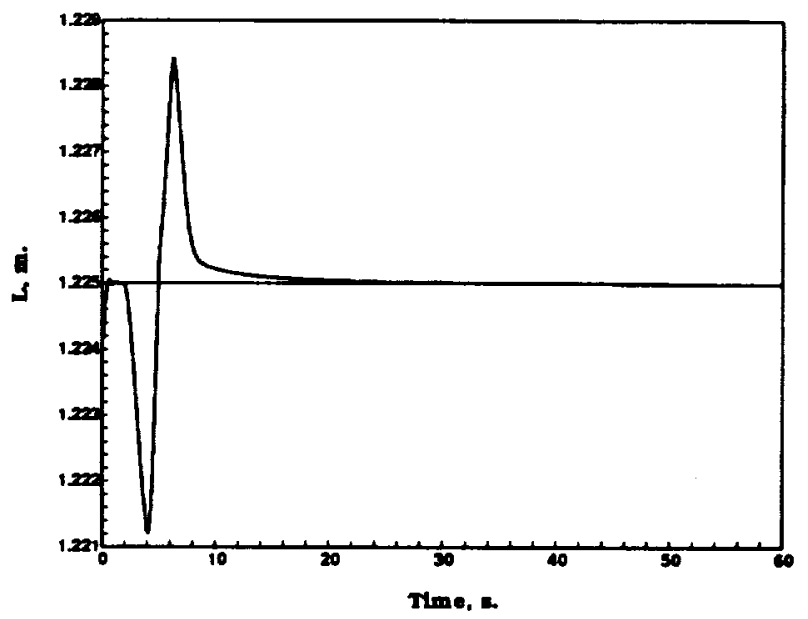

Figure 7.- Axial extension, L, caused by the EPCU versus time.

Compare the vibratory motion of the wrist (figure 8) with the smooth motion of ABV1 (see figure 9). These figures illustrate that stable operation of the EPCU can be achieved and that vibratory wrist motions are not transmitted to the payload.

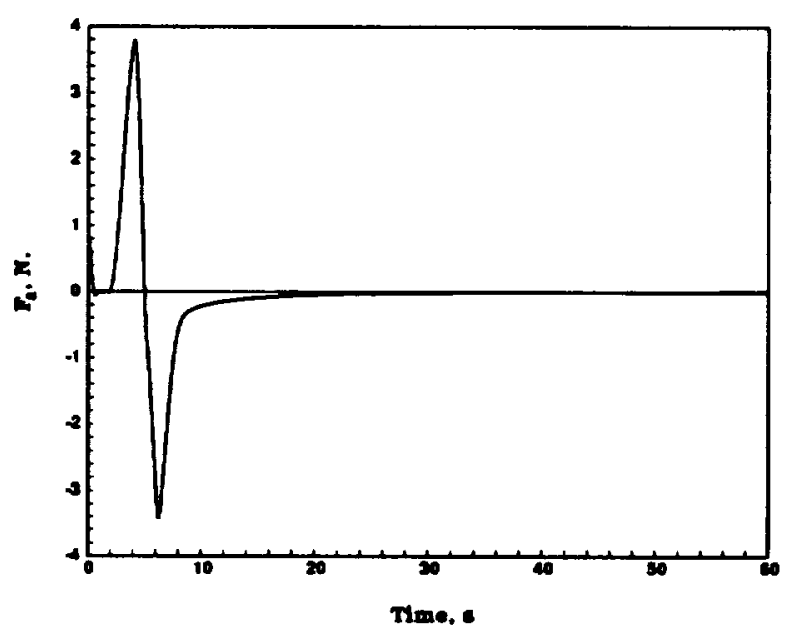

Figure 6.- Force, $F_{\mathrm{a}}$, transmitted to $A B V 1$ versus time.

\section{EPCU Test Setup and Procedure}

The EPCU was integrated into the interim testbed by mounting it between the wrist joint motor and ST. 


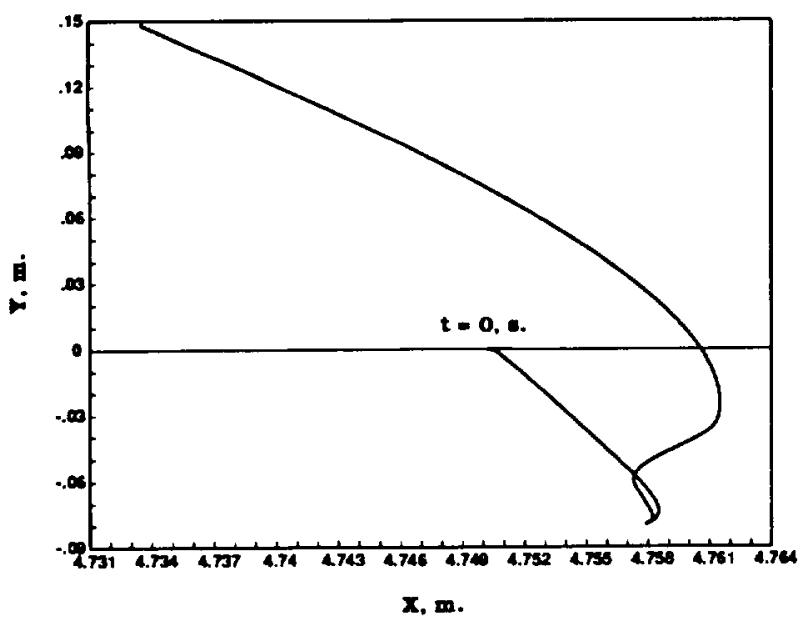

Figure 8.- Planar motion of the arm wrist.

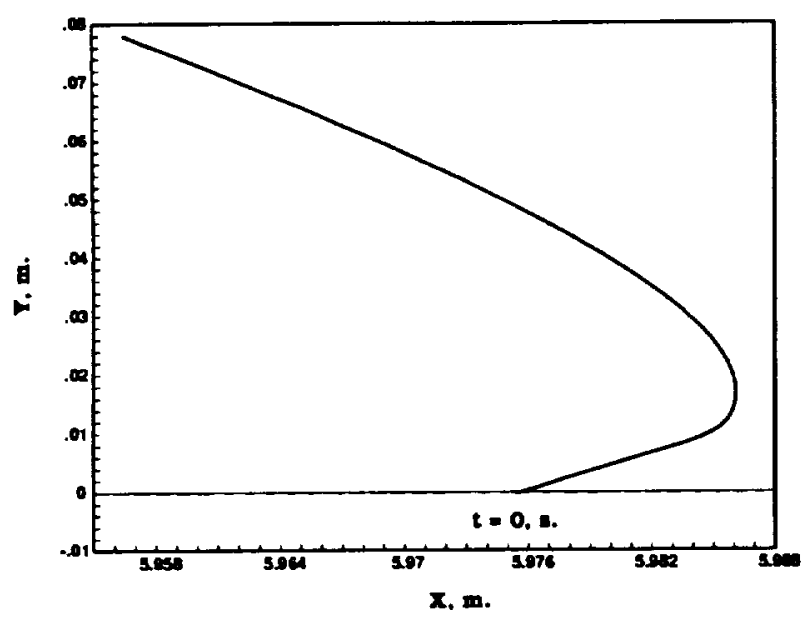

Figure 9.- Planar motion of the center of gravity of the payload, ABV1.

An air pad was added at the wrist joint so that the EPCU did not support the weight of the joint and arm.

The computer onboard ABV1 operated independently from the EPCU controller. Therefore, a digital output was provided to the EPCU controller to allow the computers to be synchronized at the start of runs. Additional analog outputs were added to the ABV1 onboard computer so that velocity commands for $x$ and $y$, and angular velocity commands about $z$ could be communicated to the EPCU controller.

The precision positioning mode of operation of the EPCU was tested by commanding the joints of the manipulator arm to lock at constant position and then commanding the EPCU to move the payload from the parked position, the extreme end of the EPCU deflection range, to the center of the deflection range, a distance of $0.0254 \mathrm{~m}$ in approximately 2 seconds. This mode is primarily for teleoperation and was not studied in detail since the testbed does not support the displays and controls needed for that evaluation. The dynamic interaction of the EPCU with the manipulator and the payload was studied in detail by using a fully automated maneuver sequence.

While developing and testing the EPCU controllers, many runs were made while varying parameters such as arm retraction rate and retraction distance. The testbed allowed baseline runs to be easily obtained by rigidly clamping the EPCU at its center position.

For each of the tests, the initial configuration of the testbed was as shown in figure 5. For each of the active EPCU tests, prior to the start of the run the EPCU was commanded to center position from the parked position used for stowage. Data was not taken during this portion of the maneuver during which the payload moved $0.0254 \mathrm{~m}$ in approximately 2 seconds and the joint motors of the manipulator were commanded to hold at the initial configuration. At the start of each run the arm was commanded to hold stationary for 5 seconds and then it was commanded to retract at a constant rate along the negative $x$-axis (figure 5). The distance commanded to retract was $0.305 \mathrm{~m}$ for short runs and $2.134 \mathrm{~m}$ for long runs, and the constant retraction rate varied between 0.0244 and $0.0457 \mathrm{~m} / \mathrm{s}$. The retracted position was held for 10 seconds and then the arm was commanded to return to the initial position at the same rate at which it was retracted. The final position was again held for 10 seconds. Data gathering was terminated and the EPCU was commanded to return to the parked position. For long runs the arm was retracted to the maximum retraction configuration shown in figure 10 and then returned to the initial configuration shown in figure 5. The long runs moved the arm through most of its useful configurations.

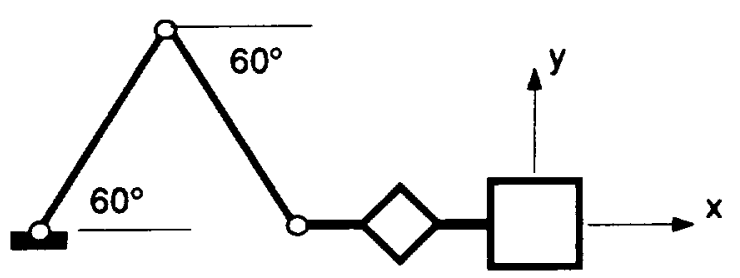

Figure 10.- Maximum retraction configuration for long test runs.

The arm controller used an inverse kinematics control scheme. The desired position of the three joints were computed from the commanded $x, y$ and theta using inverse kinematics. A torque command to each of the joints was then computed using a proportional/estimated derivative (PED) control law with position feedback coming from resolvers on each of the joints. The PED controller used a derived rate feedback obtained from a discretized washout filter differentiator with an analog transfer function, 
$\tau S /(1+\tau S)$. Experiments were performed to determine the best time constant in the differentiator based on obtaining the highest gain on the rate term of the PED controller. The value of $1 / 20$ second was selected based on these studies.

Initial testing of the EPCU was limited to a one week time period because of the expense of bringing together the equipment and personnel. During testing five control laws, briefly described below, were given a preliminary evaluation:

\section{Stiffness Controller}

The stiffness controller attempts to maintain a deflection of the EPCU which is proportional to the force feedback signal. The controller creates an error signal which is proportional to the difference between this desired deflection and the actual position of the EPCU. The error signal is then used as the velocity command to the stepper motor.

\section{"Meantz" Controller}

The "meantz" controller is identical to the stiffness controller except that the force feedback signal is conditioned by subtracting the current value from the mean value of the past $\mathbf{N}$ readings. $\mathbf{N}$ is chosen to obtain stable operation without oscillatory force output. The value of $N=75$, corresponding to a mean value time base of approximately 0.47 sec., was used. This eliminates the effects of constant offset forces, as well as eliminating the effects of drift in the force sensor reading.

\section{V-input Controller}

The v-input controller augments the stiffness controller with a feedforward signal computed from the desired velocity of the manipulator arm. This feedforward signal is a pulse in velocity and is designed to load the EPCU with the desired change in linear momentum, modulated by the peak force output of the EPCU motor.

\section{Bandpass Controller}

The bandpass controller attempts to minimize the force between the EPCU and the payload. The force feedback signal is conditioned using a bandpass filter and, thus, the EPCU will only react to minimize forces inside the bandpass frequencies. The reaction to the conditioned force feedback is similar to that of the stiffness control.

\section{Fuzzy Logic Controller}

The fuzzy logic controller also attempts to minimize forces between the EPCU and the payload. It uses a fuzzy logic algorithm to compute the velocity command to the stepper motor from the feedback signals from the force/torque sensor and the motor encoder. The fuzzy logic controller was developed using AllenBradley's Fuzzy Logic Explorer (A-B FLEX), a software application for the development of fuzzy logic control systems (see reference [9]).

\section{Results and Discussion}

Results from the testing of the EPCU at the Langley/Marshall flat-floor testbed in May 1994 will now be presented and discussed. The precision positioning mode of operation, wherein the manipulator joints are held at a constant position and the EPCU conducts fine positioning maneuvers, was studied by observation during the unparking and parking maneuvers. During these maneuvers no adverse dynamic interaction problems were observed with the manipulator. Further evaluation of this mode has been deferred until a telerobotic capability is added to the testbed. The remaining discussion pertains to fully automated studies of the isolation and load control capabilities of the EPCU. Results are presented and discussed for the case of the EPCU inactive and locked, referred to hereafter as the clamped case, and for the cases corresponding to the five controllers.

Figure 11 displays data from the clamped case test run where the arm moves at a velocity of 2.44 $\mathrm{cm} / \mathrm{sec}$. (the design speed) a distance of $0.3048 \mathrm{~m}$ in the direction of EPCU actuation, and then moves back to its starting position. In this test run, the EPCU is clamped at its center position. The results show the behavior of the arm controller attempting to follow a smooth trajectory. The peak-to-peak force for this test run is $96.52 \mathrm{~N}$. Figure 12 shows the EPCU with the stiffness controller, which uses force and position feedback from the unit itself to react as a linear spring, in this case with a stiffness of $30.65 \mathrm{~N} / \mathrm{cm}$. Although the position tracking performance during the constant velocity segments of the motion is similar for these two cases, the transient behavior at discontinuities in velocity is much more pronounced for the stiffness controller. For this test run, the peak-to-peak forces have been reduced to $66.72 \mathrm{~N}$. This increases payload overshoot during stopping and starting but reduces peak loads. 


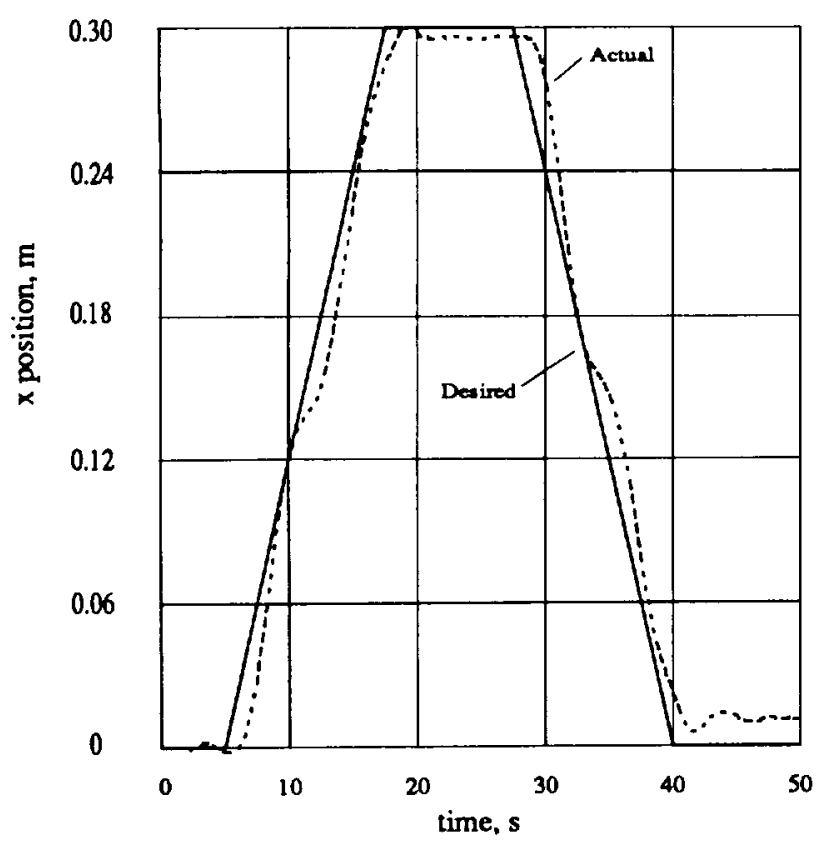

Figure 11.- Tracking performance with the EPCU inactive.

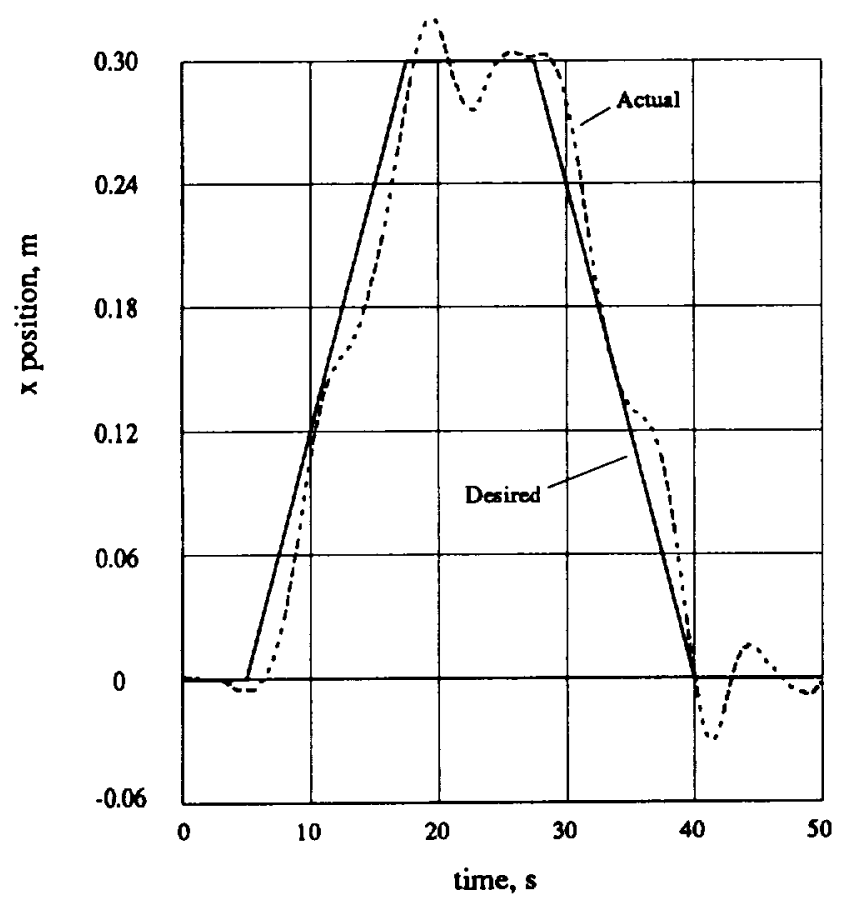

Figure 12.- Tracking performance of the EPCU with the stiffness controller.

Figure 13 illustrates the results of the EPCU running with the velocity input controller. The controller uses not only force and position feedback from the EPCU to control its position, but also uses the commanded velocity of the manipulator arm as a feedforward signal to offset the transient effects. The results show slightly better tracking ability than the clamped case as well as much improved transient behavior over the stiffness controller during starting and stopping. The peak forces here are design parameters and were allowed to return to their original (clamped) level.

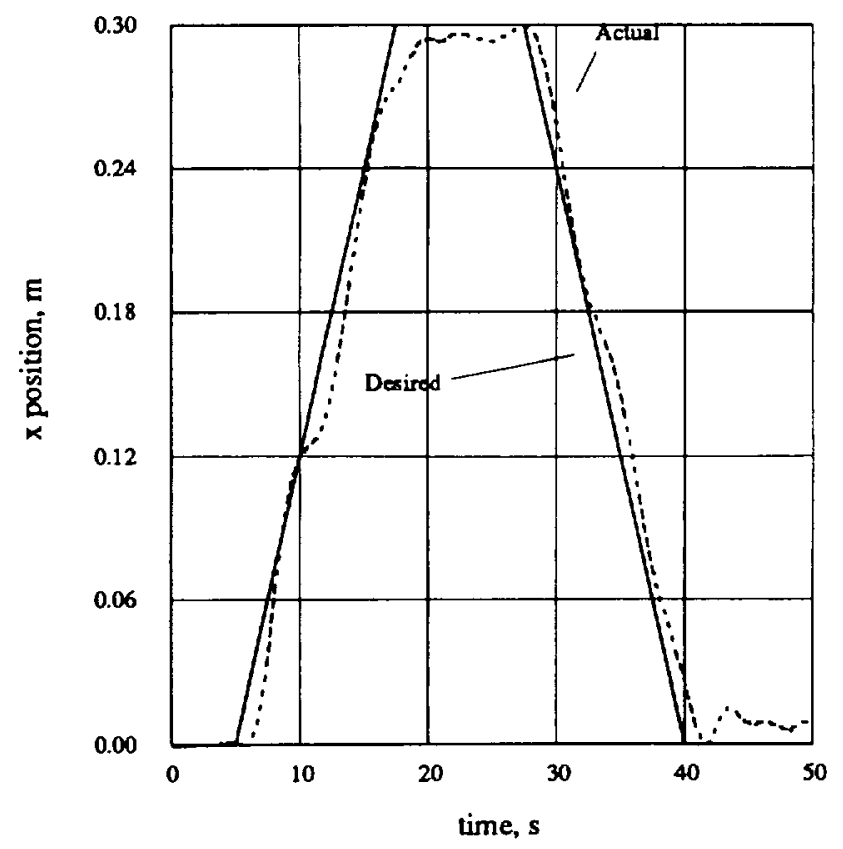

Figure 13.- Tracking performance of the EPCU with velocity input controller.

Figures 14 and 15 are difficult to compare with the earlier results, as the manipulator arm in this case moves at a speed of $4.57 \mathrm{~cm} / \mathrm{sec}$, causing higher forces between arm and payload. However, they have been included here to illustrate the behavior of two other controllers developed for the EPCU. Figure 14 shows the results of the bandpass controller. The results show very smooth velocity tracking, although, without any feedforward signal, the transients are again large. Figure 15 shows the results of a fuzzy logic controller, which again shows smooth velocity tracking but large transients. The peak-to-peak forces for these two controllers are almost equal. At the time of writing results of peak-to-peak forces for the clamped EPCU at this speed are not available.

Thus far, the discussion has been limited to short runs wherein transient forces due to velocity discontinuities can be highlighted. Now, consider longer runs and compare the performance with different controllers relative to tracking and interface loads. The long runs correspond to maneuvers between the initial configuration shown in figure 5 and the configuration of figure 10. Three sets of long runs were made corresponding to speeds of $2.44,3.66$, and $4.57 \mathrm{~cm} / \mathrm{s}$. The initial segment of each run was a commanded retraction of the payload at constant speed to the 


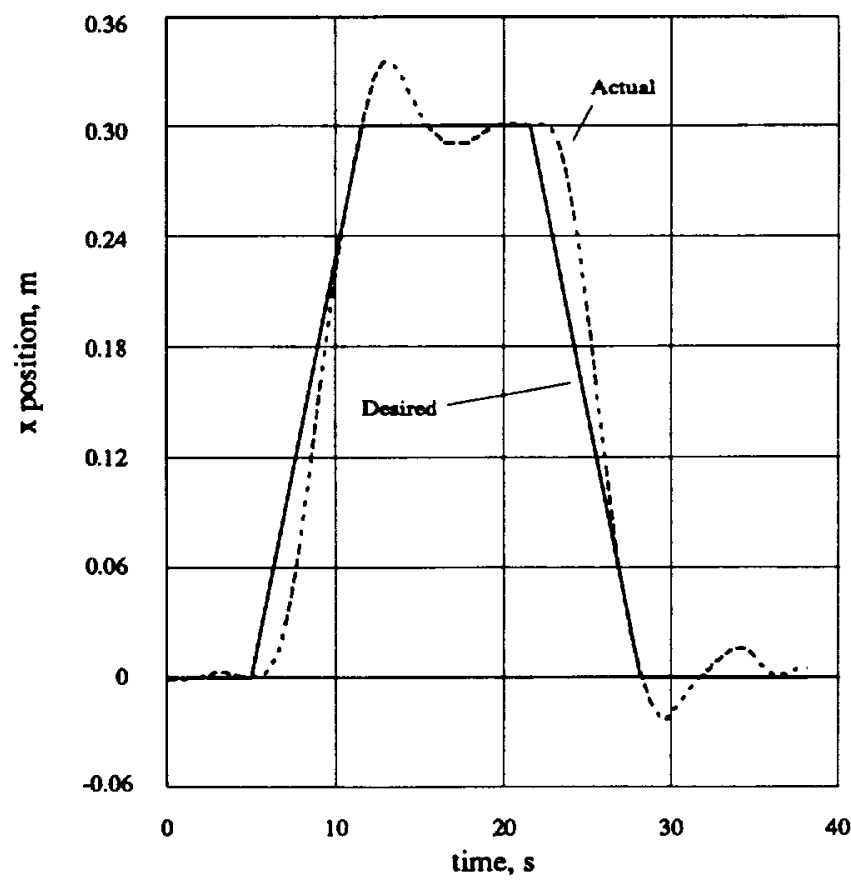

Figure 14.- Tracking performance of the EPCU with the bandpass controller.

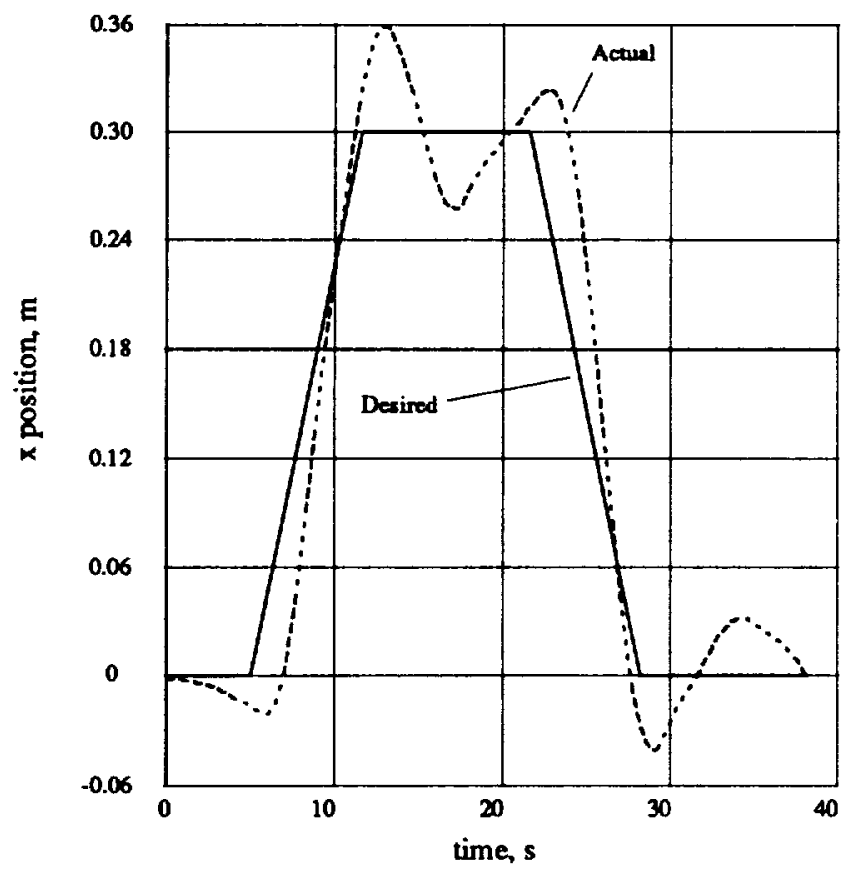

Figure 15.- Tracking performance of the EPCU with a fuzzy logic controller.

configuration of figure 10. The time for this segment is, of course, speed dependent. For all long runs this segment was followed by a position-hold command segment for 10 seconds, an extension segment , and another position-hold command segment for 10 seconds.

The first set, corresponding to the design speed of $2.44 \mathrm{~cm} / \mathrm{sec}$, requires a time for retraction of 87.5 seconds. The tracking performance for the clamped case is illustrated in figure 16 and that of the "meantz" case is illustrated in figure 17. The standard deviation, $\sigma$, of the $x$ displacement error is $0.02527 \mathrm{~m}$ for the "meanfz" control law case, $0.02601 \mathrm{~m}$ for the clamped case, and $0.02762 \mathrm{~m}$ for the stiffness control law case. The standard deviation for the "meanfz" case was the lowest of all cases. Also, for this case, the response tracks the command very closely especially in the final segment with position-hold commands. For the stiffness case, however, there was a degradation of position tracking error over the clamped case. Concerning interface loads, one might expect a reduction of peak load corresponding to an increase in tracking error. Unfortunately, the load cell data for this clamped case run were corrupted so that the conjecture cannot be verified. For the two active control laws being discussed, the stiffness control law has smaller peak loads, $55 \mathrm{~N}$, than the "meantz" control law, $70 \mathrm{~N}$.

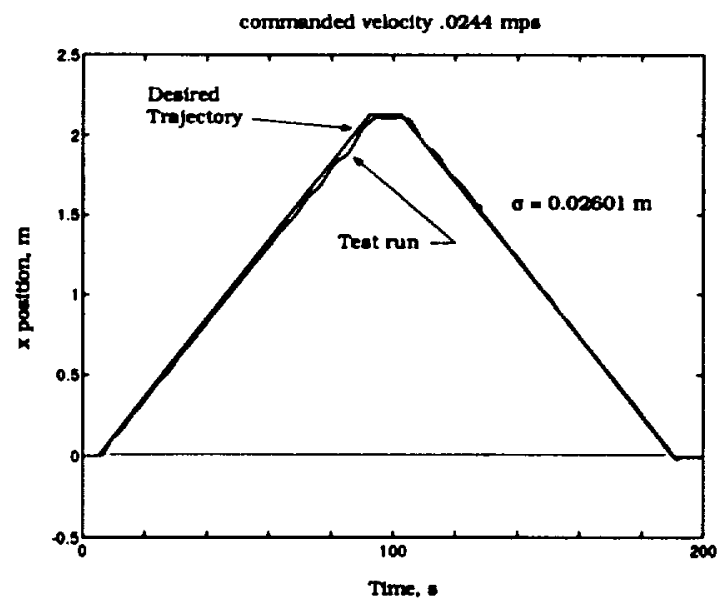

Figure 16.- Tracking performance of the manipulator with the EPCU clamped for a long run.

In the second set of long runs the speed of retraction and extension was increased to $3.66 \mathrm{~cm} / \mathrm{s}$ and the time was reduced to $58.25 \mathrm{~s}$ for the retraction and extension segments. The standard deviation of the $x$ position error was $0.02969 \mathrm{~m}$ and peak force was $45 \mathrm{~N}$ for the $v$-input controller. For the clamped case it was $0.03005 \mathrm{~m}$ and peak force was $75 \mathrm{~N}$. This shows that the EPCU is capable of increasing the precision of tracking with simultaneous load reduction.

In the third set of long runs the speed of retraction and extension was increased to $4.57 \mathrm{~cm} / \mathrm{sec}$. reducing the time of the retraction and extension segments to $46.6 \mathrm{sec}$. The standard deviation of the $x$ position 


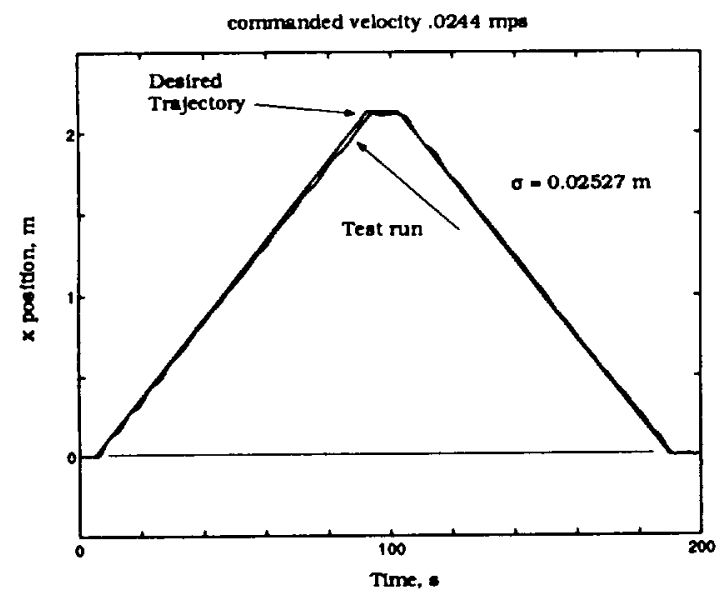

Figure 17.- Tracking performance of the EPCU with the "meanfz" controller for a long run.

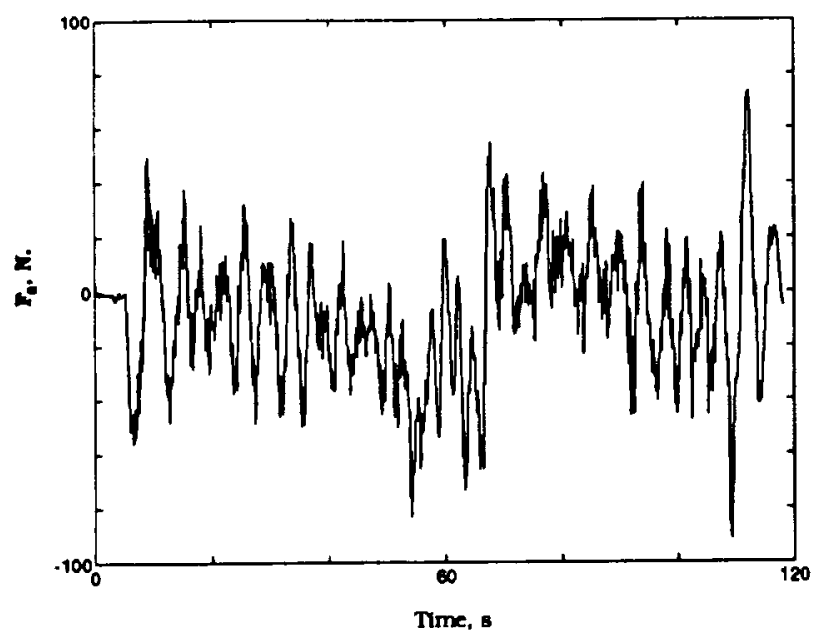

Figure 18.- Load, $F_{2}$, transmitted to the payload for the clamped case at $4.57 \mathrm{~cm} / \mathrm{s}$.

error was $0.03123 \mathrm{~m}$ and peak force was $90 \mathrm{~N}$ for the $v$-input controller. For the clamped case the standard deviation in position error was $0.03399 \mathrm{~m}$ and peak force was $75 \mathrm{~N}$. This shows that, for this set of runs, the EPCU with a v-input control law improves position tracking at the expense of increasing peak forces. It should be noted, however, that this force increase occurs during starting and stopping the payload at fullest extension of the manipulator for the run. The forces transmitted ignoring these initial transients are actually lower for the $v$-input case than for the clamped case. This can be seen by comparing figure 18 and figure 19. It is possibly caused by the v-input impulse mechanization, i.e. a constant, maximum amplitude force command for the time required to generate the payload velocity change, which is expected to be more critical at higher speeds. A redesign of the impulse shape could possibly reduce these peak forces.

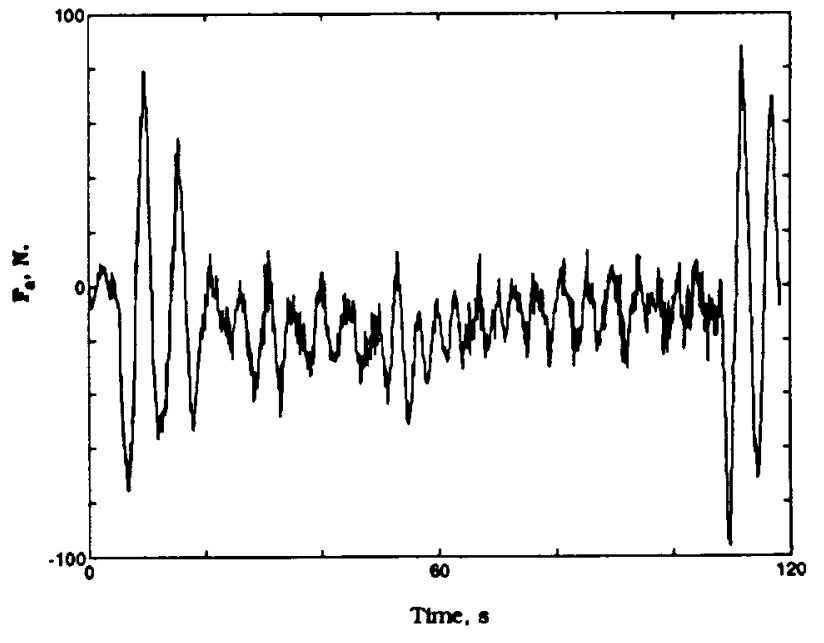

Figure 19.- Load, $F_{a}$, transmitted to the payload for the $v$-input case at $4.57 \mathrm{~cm} / \mathrm{s}$.

\section{Concluding Remarks}

From the limited testing and simulation studies accomplished to date on the EPCU, the following conclusions can be reached:

1. Concerning the precision positioning mode of operation, no adverse dynamic interaction was observed between the manipulator arm and the EPCU in parking and unparking the payload.

2. Simulation results have shown that stable operation of the EPCU can be achieved and that vibratory wrist motions are not transmitted to the payload.

3. The EPCU has successfully reduced the loads transmitted to the payload by $31 \%$ using the stiffness controller and up to $40 \%$ using the v-input controller.

4. Tracking performance was improved from an $x$-displacement tracking error of $0.02601 \mathrm{~m}$ (EPCU clamped) to $0.02527 \mathrm{~m}$ ("meanfz" case) at the design speed of $2.44 \mathrm{~cm} / \mathrm{sec}$.

5. During testing, the EPCU did not experience deflection saturation during runs with speeds up to 1.87 times the design speed for any of the control laws tested. The margin was approximately \pm .5 $\mathrm{cm}$ out of the total operating range of $\pm 2.54 \mathrm{~cm}$.

The capture and berthing of a controlled spacecraft using a robotic manipulator is an important technology for future space missions that require precision telerobotic maneuvering of large payloads by the astronauts using the Remote Manipulator System (RMS) of the Space Shuttle. Since the RMS was not designed with this in mind, augmentation of the system may be needed. This paper has proposed an augmentation that will allow precision payload positioning and active monitoring of loads transmitted 
to the payload. The potential solution proposed herein, which is intended to solve the problem with little or no impact on the existing RMS, involves an independent device called an End-Point Control Unit (EPCU) that interfaces between the payload and the end-effector of the RMS and actively controls loads transmitted to the payload using active compliance and/or inertial load relief.

The specific device studied herein is a single degree-of-freedom, active compliance device using a stepper drive motor attached to a rack and pinion drive and a load cell. Results of simulation studies designed to size the unit for test on a testbed at the Marshall Space Flight Center, MSFC, have been presented, as have been results of the initial test activity at MSFC. The results and conclusions presented above show promise, although not conclusive evidence, that the EPCU concept can improve the performance of a flexible manipulator relative to both precision tracking and interface loads. Concerning the stiffness control law, two points can be made. First, we can reduce the forces transmitted to the payload at the expense of positional accuracy. Second, and visa versa, we can increase positional accuracy at the expense of forces transmitted to the payload. The $v$-input control law was successful at simultaneously improving the tracking performance and interface load reduction. With more testing, all control laws described above can be modified to include feedforward signals as in the existing, proactive, v-input controller with the hope that tracking errors and loads can simultaneously be reduced.

We note several improvements that can be made to the EPCU as a result of the experience gained. One fundamental problem is that the EPCU essentially uses force as its primary feedback for control. For the unit tested, the primary output command of the EPCU control computer is velocity of the stepper motor. Force is only indirectly commanded. It is believed that the use of a direct drive DC motor with a high bandpass electric current amplifier will improve the performance and lower the noise experienced in the load cell output. Another problem experienced by the v-input controller is that, because of unmodelled friction in the facility, the payload slows down following a force pulse. This degrades the $v$-input controller tracking performance since no direct measurement of payload velocity is available. The EPCU can be instrumented with inertial quality accelerometers, which can be used to compute the velocity of the payload and use this signal as the primary EPCU feedback.

\section{References}

[1] R. H. Cannon and E. Schmitz, Initial Experiments on the End-Point Control of a Flexible One-Link Robot. Int. J. of Robotics Research, Vol. 3, No. 3, Fall 1984, pp. 62-75.

[2] M. Timmerman and S. L. Dickerson, High Bandwidth Control of Flexible Robots: Proof of Concept Experiments for Momentum Management. Proc. American Control Conference, May 1990, pp. 200-204.

[3] R. C. Montgomery, D. Ghosh, and S. P. Kenny, Analytic and Simulation Studies on the Use of Torque-Wheel Actuators for the Control of Flexible Robot Arms. Advances in Dynamics and Control of Flexible Spacecraft and Space-Based Manipulators, DSC-Vol. 21, November 1991.

[4] R. C. Montgomery, S. P. Kenny, D. Ghosh, and J. Shenhar, Evaluation of Inertial Devices for the Control Of Large, Flexible, Space-Based Telerobotic Arms. Proc. Fifth Annual NASADOD CSI Technology Conference, March 3-5, 1992.
[5] D. Manouchehri, T. Lindsay, and D. Ghosh, Hardware Interface for Isolation of Vibrations in Flexible Manipulators - Development and Application. Space Operations, Applications, and Research Symposium, August 3-5, 1993.

[6] R. C. Montgomery, P. A. Tobbe, J. Weathers, D. Ghosh, and J. L. Garrison, A Testbed for Research on Manipulator Coupled Active Spacecraft. AIAA Guidance, Navigation, and Control Conference, AIAA paper No. 93-3712, August 9-11, 1993.

[7] Integrated Systems, Inc., Palo Alto, California, MatrixX Core Users Manual and System Build V2.4 Users Guide, Edition 8, June 1991.

[8] B. W. Char et al., MAPLE Reference Manual, 5th Edition. Department of Computer Science, University of Waterloo, Waterloo, Ontario, CANADA N2L 3G1, March 1988.

[9] Allen-Bradley Company, Milwaukee, WI, A-B FLEX Version 2.0 User's Guide, April 1992. 
